\title{
OEIS complex: a rare foetal anomaly
}

\section{Neelamma G. Patil*, Subash R. Mudanur, Aruna S. Nemagouda, Shreedevi S. Kori, Lubna Y. Lahori}

Department of Obstetrics \& Gynaecology, BLD University’s Shri B. M. Patil Medical College, Bijapur-586103, Karnataka, India

Received: 8 July 2014

Accepted: 13 July 2014

*Correspondence:

Dr. Neelamma G. Patil,

E-mail: patilneelgiri@rediffmail.com

(C) 2014 Patil NG et al. This is an open-access article distributed under the terms of the Creative Commons Attribution Non-Commercial License, which permits unrestricted non-commercial use, distribution, and reproduction in any medium, provided the original work is properly cited.

\begin{abstract}
(Omphalocele, extrophy of bladder/cloaca, imperforate anus, spinal defects) is a rare constellation of malformations involving multiple organs in humans. It is the most serious manifestation of exstrophy-epispadias sequence. The exact aetiology of this condition is not known. It is sporadic but genetic associations have also been hypothesised. It results from defective blastogenesis leading to improper closure of anterior abdominal wall and defective development of cloacae and urogenital septum. Incidence ranges from 1 in 200000 to 1 in 400000 . Prenatal diagnosis of OEIS complex can be done by anomaly scan between 18 to 22 weeks of gestation. It carries a poor prognosis. Survival is nil or very less. Even if baby survives it requires multiple surgeries with many potential complications. We had an undiagnosed case of this complex presenting to us in advanced labour, but a timely ultrasound helped in the diagnosis and avoided an unnecessary caesarean section which we would have done for an elderly primigravida with breech presentation.
\end{abstract}

Keywords: OEIS complex, Foetal anomaly, Anomaly scan

\section{INTRODUCTION}

OEIS complex is a rare constellation of congenital foetal malformations like omphalocele, exstrophy of bladder/cloacae, Imperforate anus and spinal defects. ${ }^{1}$ It is the most serious manifestation of exstrophy-epispadias sequence. $^{2}$

The exact aetiology of this condition is not known. ${ }^{3}$ It is sporadic but genetic associations have also been hypothesised. It results from defective blastogenesis leading to improper closure of anterior abdominal wall and defective development of cloacae and urogenital septum. $^{2}$ Incidence is estimated to be 1 in 200000 to $400000 .^{4}$

We present this rare case which was not diagnosed antenatally (as patient had not taken antenatal care) and presented to us in second stage of labour.

\section{CASE REPORT}

A 30 year, primigravida, with non-consanguineous marriage with spontaneous conception after 7 years of married life, presented to our hospital with labour pains. She had not taken any antenatal care and no antenatal scans were done. There was no history of diabetes or any other medical disorder or intake of teratogenic drugs, no family history of congenital malformations. She had history of cleft lip which was repaired in her early childhood. She had no history of smoking nor addicted to alcohol.

On examination; uterus was 32 to 34 week size, acting, with breech presentation, FHS was 136 bpm. On vaginal examination, cervix was fully dilated with presenting part (breech) at - 2 station and pelvis was adequate. Haematological and biochemical parameters were normal. 
With the view that breech presentations are usually associated with anomalies and she was elderly primigravida, an emergency scan was done. It revealed a single live intrauterine foetus of gestational age of 34 weeks 2 days with breech presentation with evidence of sac measuring $15 \times 21 \mathrm{~mm}$ size in lumbo sacral region with no evidence of posterior elements (meningocele/meningomyocele). Other anomalies could not be made out due to severe oligo hydramnious (?leak). The prognosis was explained to patient's family and they opted for vaginal delivery. Assisted breech delivery was conducted and a still born baby with multiple anomalies was delivered. Autopsy of the baby was done.

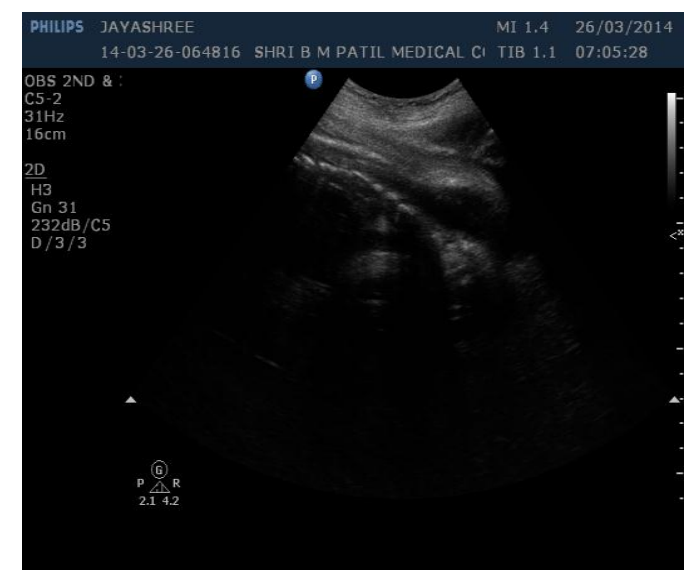

Figure 1: Meningocoele measuring $15 \times 21 \mathrm{~mm}$ in size in lumbo sacral region.

\section{Autopsy report}

External examination: A pre-term baby corresponding to 32-34 weeks of gestational age with weight of $2 \mathrm{~kg}$ and length $50 \mathrm{~cm}$. A large ompohalocele $(10 \times 8 \mathrm{~cm})$ arising from abdominal wall defect was seen at level of pubic symphysis. Right foot congenital talipus equinovarus was seen. Scrotal sac was well developed to age but widely placed; testicals were not seen in scrotal sac. Phallus was underdeveloped with distinct fold of prepuce and glans, anal opening was absent in perineal region. A soft swelling of $6 \times 8 \mathrm{~cm}$ was seen in gluteal region.

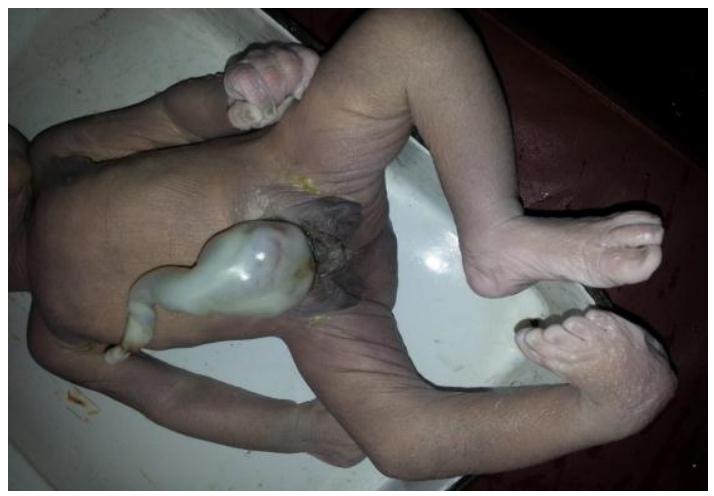

Figure 1: Photograph showing omphalocele, imperforate anus, right foot CTEV.

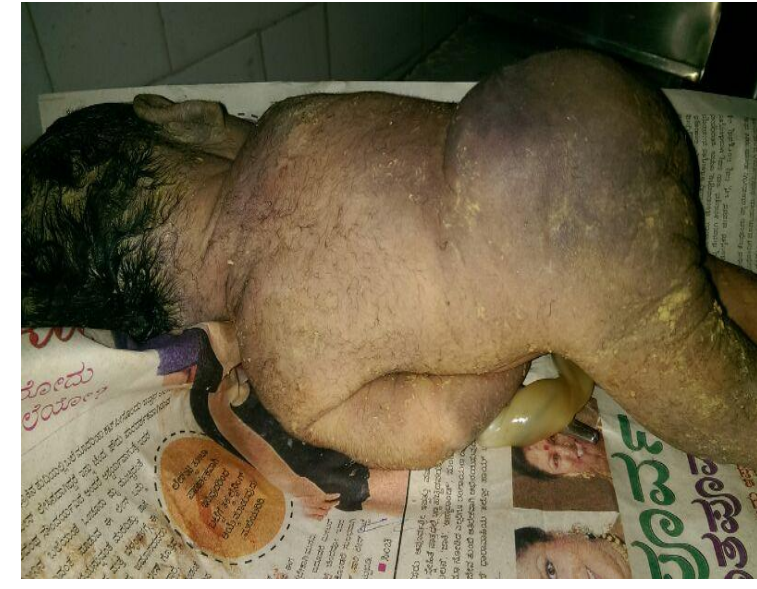

Figure 3: Soft swelling of $6 \times 8 \mathrm{~cm}$ in gluteal region with meningocele.

Internal examination: Thoracic cavity was normal. The liver was situated in left hypochondriac region. Most of the coils of intestine were protruding into umbilical cord through anterior abdominal wall defect (omphalocele). Spleen, pancreas, kidneys, adrenal glands and bladder were absent/hypoplastic, right dome of diaphragm was also not seen. Small intestine continued into muscular sac and ended blindly. Bilaterally hypo plastic gonads (testis) were found in ileac fossa.

\section{DISCUSSION}

OEIS complex - omphalocele, exstrophy of bladder/cloaca, imperforate anus, spinal defects are rare constellation of malformations involving multiple organs in humans. It is a clinically recognised non-random association that, in addition to four classic malformations is variably associated with other anomalies like spina bifida, genital abnormalities, renal malformations, symphysis pubis diastasis, GIT malformations and limb abnormalities. ${ }^{3}$ Male:female ratio is $2: 1 .^{5}$

First description of OEIS complex case was published by Litter et al. in $1709 .{ }^{6}$ It was previously called as exstrophy of cloaca, vesico-intestinal fissure, exstrophy splanchana. The term OEIS complex was first used by Carrey JC et al. in $1978 .^{7}$

Most of the cases are sporadic with no obvious aetiology. There is a higher concordance of OEIS in monozygotic twins than in dizygotic twins suggesting a possible genetic contribution. ${ }^{1}$ It is also reported in siblings from separate pregnancies. ${ }^{6}$ And in patients with family history of similar malformations (bladder exstrophy, epispadiasis imperforate anus. ${ }^{8}$ The complex is also been reported in association with trisomy 21 , trisomy 18 , triple-X syndrome, turner's mosaicism and 1p 36 deletion. ${ }^{1}$ Christopher et al. conducted genetic analysis of 13 cases of OEIS complex and did not find any disease causing mutations, although novel variants were identified in five patients. ${ }^{1}$ 
Cloacal exstrophy in humans has reported in association with maternal exposure to diphenyl hydantoin, diazepam, valproic acid, methamphetamine, cigarette smoking, maternal obesity and diabetes mellitus, but all these associations are limited to 1-2 cases and there is insufficient data to link OEIS complex to any specific teratogens. Cloacal exstrophy is reported in infants who were conceived by in-vitro fertilisation and it was suggested that ex vivo manipulation of embryo or altered biochemical milieu of IVF pregnancies may affect embryogenesis and lead to such anomalies. ${ }^{3}$

In humans cloaca is a phylogenetic embryonic structure, where caudal parts of digestive, genital and urinary systems join. Normal development gives rise to lower abdominal wall with bladder, intestine, anus, genital organs, pelvic bones and lumbo-sacral spine. OEIS complex results from defect in early blastogenesis or a defect of caudal mesodermal migration during primitive streak period that later contributes to the formation of infraumbilical mesenchyme, cloacal septum and vertebrae. These defects lead to improper closure of ventral abdominal wall due to failure of convergence of four ecto-mesodermal folds (a cranial, a caudal and two lateral) of embryo with associated defects in development of cloaca and urorectal septum during $4^{\text {th }}$ week and nonreduction of physiological hernia and non-fixation of gut between $8^{\text {th }}-12^{\text {th }}$ weeks of development. Lack of mesoderm in the infra umbilical abdominal wall result in omphalocele. Cloacal exstrophy prevents the development of proctodeum resulting in imperforate anus. Caudal dysgenesis interferes with somite formation resulting in defective vertebrae. ${ }^{2}$

Cases of OEIS complex show features overlapping or associated with other syndromes affecting caudal region of the embryo, such as, Sirenomelia, VACTERL (Vertebral, Anal, Cardiac, Trecheo-Esophageal fistula, Renal and Radial, Limb anomalies), CRS (Caudal Regression Syndrome), URSMS (Uro-Rectal Septum Malformation Sequence), LBWC (Limb Body Wall Complex). ${ }^{4}$

Prenatal diagnosis of OEIS complex can be done by anomaly scan between 18 to 22 weeks of gestation based on following features, non-visualisation of foetal bladder, infra umbilical anterior wall defect, omphalocele, myelomenigocele and, minor criteria like lower extremities anomalies, ascites, widened pubic arches, kyphoscholoisis, hydrocephalus and single umbilical artery. $^{9}$

In our case, patient had not taken any antenatal care; hence this anomaly was not diagnosed in early stages by ultrasound (USG). She reported to our hospital only in second stage of labour.

Since it was a precious pregnancy with breech presentation, normally we would have done an emergency Caesarean Section (CS). But timely decision of an emergency USG prevented the CS \& its associated complications.

Compatibility of life with OEIS complex is nil or very less. Even if baby survives it requires multiple surgeries with many potential complication including renal, reproductive, ambulatory impairments and psycho social defects. In absence of open neural tube defects brain is structurally and functionally normal and normal cognitive development can be expected. ${ }^{10}$

\section{CONCLUSION}

OEIS complex is a rare congenital malformation of unknown aetiology, with poor prognosis. Early prenatal diagnosis by anomaly scan can give an option to parents for termination of pregnancy and also help to plan appropriately for perinatal management in less severe cases.

\section{Funding: No funding sources Conflict of interest: None declared Ethical approval: Not required}

\section{REFERENCES}

1. Christopher N. Vlangos, Amanda Siuniak, Todd Ackley, Hans Van Bokhove, Joris Veltman, Ram Iyer, et al. Comprehensive genetic analysis of OEIS complex reveals no evidence for a recurrent microdeletion or duplication. Am J Med Genet Part A. 2001;155:38-49.

2. Subhatra Devi V, Md. K. Faheem N, Vidyavathi Ch. Usharani V. OEIS complex: a rare case report. Int $\mathbf{J}$ Res Dev Health. 2013;1(1):21-5.

3. Ayman W. El-Hattab, Josh C. Skorupski, Michael H. Hsieh, Amy M, Sau Wai Cheung. OEIS complex associated with 1p 36 deletion. Am J Med Genet Part A. 2009;152A:504-11.

4. Sunita Arvind Athavale. Deciphering caudal embryonic defects: embryological analysis and reviewing literature data. Rom J Morphol Embryol. 2012;53(4):1013-20.

5. Martinez Frias ML, Bermejo E, Rodriguez-Pinilla E, Frias JL. Exstrophy of cloaca and exstrophy of bladder. Two different expression of primary developmental field defect. Am J Med Genet. 2001;99:261-9.

6. Smith NM, Cambers HM, Furness ME, Haan EA. The OIES complex recurrence in sibs. J Med Genet. 1992;29:730-2.

7. Carey JC, Greenbaum B, Hall BD. The OEIS complex (omphalocele, exstrophy, imperforate anus, spinal defects). Birth Defects. 1978;14:253-63.

8. Keppler Noreuril KM. OEIS Complex (omphalocoele-exstrophy-imperfrote anus-spinal defects): a review of 14 cases. Am J Med Genet. 2001;99-271-9. 
9. Noack F, Sayk F, Gembruch U. Omphalocele exstrophy imperforate anus defects complex in dizygotic twins. Foetal Diagn Ther. 2005;20:346-8.

10. Tiblad E, Wilson D, Carr M, Flake A, Hedrick H, Johnson M, et al. OEIS sequence: a rare congenital anomaly with prenatal evaluation and postnatal outcome in six cases. Prenatal Diagn. 2001;28:141-7.

DOI: $10.5455 / 2320-1770 . i j r \operatorname{cog} 20141244$

Cite this article as: Patil NG, Mudanur SR, Nemagouda AS, Kori SS, Lahori LY. OEIS complex: a rare foetal anomaly. Int J Reprod Contracept Obstet Gynecol 2014;3:1100-3. 